Article

\title{
Electrically Tunable-Focusing Liquid Crystal Microlens Array with Simple Electrode
}

\author{
Li-Lan Tian ${ }^{1}$, Fan Chu ${ }^{2}, \mathrm{Hu}$ Dou ${ }^{2}$, Lei $\mathrm{Li}^{2}$ and Qiong-Hua Wang ${ }^{1, *}$ \\ 1 School of Instrumentation and Optoelectronic Engineering, Beihang University, Beijing 100191, China \\ 2 School of Electronics and Information Engineering, Sichuan University, Chengdu 610065, China \\ * Correspondence: qionghua@buaa.edu.cn; Tel.: +86-010-8233-8326
}

Received: 15 July 2019; Accepted: 16 August 2019; Published: 20 August 2019

\begin{abstract}
An electrically tunable-focusing liquid crystal (LC) microlens array exhibiting a wide-range tunable focal length is proposed. The lower substrate has strip indium tin oxide (ITO) electrodes, the upper substrate has periodic ITO electrodes with a certain gap coated on the inner surface., and an LC microlens is generated between the two strip electrodes. For each LC microlens, the gap between the top planar electrodes is directly above the center of the microlens. Unlike the conventional LC lens, the individual LC microlens is not coated with ITO electrodes on the central part of its upper and lower substrates, which helps to maintain the LC's horizontal orientation. In the voltage-off state, the focal length of the microlens array is infinity because of the homogeneous LC alignment. At a given operating voltage, an ideal gradient refractive index distribution is induced over the homogeneous LC layer, which leads to the focusing effect. The simulation result shows that the focal length of the LC microlens could be gradually drawn to $0.381 \mathrm{~mm}$ with a change of voltage.
\end{abstract}

Keywords: liquid crystal; simple electrode; microlens array; wide focusing range

\section{Introduction}

A microlens array is a key part for optical information processing, optical interconnection, optic fiber switches [1-4], ophthalmic application [5], three-dimensional display systems [6-13], and so on. A tunable LC microlens array has been investigated for 40 years. For an LC microlens, in order to adjust the focal length, a spatial axisymmetric distribution of the refractive index in the LC bulk can be generated and varied by an external electric field. As there is no mechanical motion, this will facilitate a well-knit system.

To obtain a LC lens, various devices such as a multi-strip or hole-patterned electrode [8,14-17], LC lens with switchable positive and negative focal lengths [18], optically hidden dielectric structure [19,20], and polymer-stabilized blue phase LC [21-25] have been studied in recent years. Among these attempts, the multi-strip electrode structure could generate a spherical phase profile, but the driving mode is very complicated by individually addressing the pixelated electrodes. The hole-patterned electrode could obtain a large aperture LC lens to achieve good focusing spot, however, the LC layer must be very thick, which will greatly increase the response time since the response time depends on the square of the thickness of the LC layer [26-28]. In addition, the middle part of the phase profile of the hole-patterned electrode structure is relatively flat. The LC lens with switchable positive and negative focal lengths is suitable for myopia-presbyopia, and multiple LC layers are adopted to increase the phase. However, using multiple LC layers will cause a certain degree of light scattering and reduce the image quality [26-28]; moreover, the operating voltage will be increased. The optically hidden dielectric structure enables finer phase control for generating a nearly ideal parabolic phase profile, however, it has Fresnel reflections between dielectric layers, which will also affect the image quality. A polarization-independent microlens array using polymer-stabilized blue phase $\mathrm{LC}$ has a fast response 
time because of the orientation of the local LC directors in the unit lattice instead of the transition between two nematic orientations of the LC directors with higher order parameters [25]. However, the focus range of this lens is very small because the maximum refractive index difference in the BPLC lens is only one-third of the birefringence to the same LC, according to the Kerr model. Therefore, one challenging task is to develop high performance tunable microlens arrays with a simple electrode, simple driving scheme, simple fabrication process, uniform LC cell gap, low operating voltage, and wide-range tunable focal length.

In this paper, we propose a LC microlens array with a simple electrode structure that behaves as a cylindrical lens. In the center of each microlens, a certain gap between the indium tin oxide electrodes on the upper glass substrate is used to control the stability of the LC director in the center of the lens. A strip ITO electrode is at the end of the microlens. For individual microlens, when there is no electric field, there is no focusing effect because of the homogeneous LC alignment. When a voltage is applied to the strip electrodes, an axisymmetric refractive index distribution will generate within the LC bulk which, in turn, results in a focusing effect. The axisymmetric refractive index distribution across the LC lens can be obtained in a relatively low voltage $\left(4.8 \mathrm{~V}_{\text {rms }}\right)$ with only one data addressing. Furthermore, the proposed model with the planar and relatively thin cell gap exhibits a uniform and relatively fast dynamic response time.

\section{Device Structure and Principle}

Figure 1 depicts the side view of the proposed LC microlens array in the voltage-off and voltage-on states. The upper substrate has periodically planar electrodes as common electrodes. Then, the upper glass substrate is coated with an alignment material to align the nematic LC director. The lower substrate is coated with periodically strip electrodes as pixel electrodes. Likewise, the lower substrate also has a thin polyimide alignment layer and rubs in the opposite parallel direction relative to the upper substrate. As a result, an LC layer with a uniform arrangement is shaped. For each LC microlens, two adjacent strip electrodes on the lower substrate act as two border electrodes. For the center of each microlens, there is an electrode clearance on the top substrate directly above the center of the two strip electrodes on the lower substrate. As depicted in Figure $1, d_{\mathrm{LC}}$ is the thickness of the nematic LC bulk, $w$ is the width of the pixel electrodes, $g$ is the clearance between common electrodes, $l$ is the space between the pixel electrodes, $s$ is the distance from the border of individual microlens to the adjacent pixel electrode, $O$ is the central point of the microlens, and $R$ is the radius of the individual microlens.

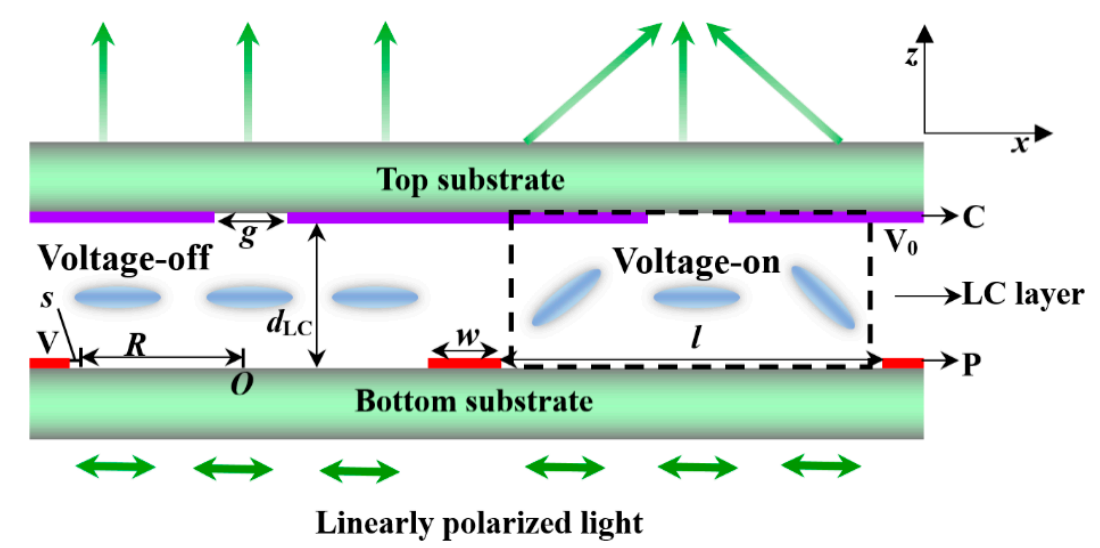

Figure 1. The configuration of the proposed liquid crystal microlens array (the area of the individual microlens is marked by a black dotted frame).

In our design, the existence of the central clearance of common electrodes has a major effect on maintaining the horizontal orientation of the LC director in the center of the microlens and the LC director tilt distribution can be adjusted by setting its width. Here, only stationary states of nematic LC cell were analyzed. In the voltage-off state, the nematic LC cell has a planar orientation with a 
homogeneous director perpendicular to the strip electrodes. When a voltage is applied to the strip electrodes, the director field of the nematic LC cell is changed by voltage and anchoring. An incident light that is linearly polarized in the rubbing direction of the cell undergoes a non-uniform phase retardation and its propagation behavior behaves as a positive cylindrical lens, as shown in Figure 1 . For the proposed LC microlens, due to the planar LC layer, flat electrodes and flat substrates, the focal length tuning range is relatively wide as it has almost no initial focusing ability. Unlike an LC lens with flat glass substrates and a curved electrode $[29,30]$, the thickness of the top and bottom substrates of the proposed model cannot shield any voltage from the LC layer, so the operating voltage of this microlens will be very low. In addition, the light scattering is very small in the process of focus change.

The incident light wave linearly polarizes along the $x$-axis and passes through different areas of the LC microlens, which will undergo different optical paths. It is assumed that $\theta$ is the angle between the LC director and the incident direction of polarized light, thus the effective refractive index of the LC is given by the index ellipsoid equation:

$$
n_{e f f}(\theta)=\frac{n_{\mathrm{o}} n_{\mathrm{e}}}{\sqrt{n_{\mathrm{o}}^{2} \sin ^{2} \theta+n_{\mathrm{e}}^{2} \cos ^{2} \theta}}
$$

where $n_{\mathrm{o}}$ is the ordinary LC refractive index, and $n_{\mathrm{e}}$ is the extraordinary LC refractive index. By using the final average effective birefringence distribution, we can calculate the refractive index difference between the border and center of the individual LC microlens. Then, the focal length can be expressed using Fresnel's approximation:

$$
f=\frac{R^{2}}{2 d_{L C} \delta n}
$$

where $\delta n$ is the effective refractive index difference between the lens center and border.

\section{Simulation Results and Discussion}

To validate the design concept, we studied the optical properties of the proposed nematic LC microlens array with a simple electrode structure and compared its performance with that of a conventional thin lens. We used commercial simulation software Tech Wiz LCD 3D (Sanayi System Company., Ltd., Korea) for the electro-optic simulations. For a LC microlens, in order to obtain a short focal length for a given lens diameter and LC cell gap, we must maximize $\delta n$ as much as possible, thus we could select a LC material with a large saturation birefringence $\Delta n$. Accordingly, in the whole simulation process, we used a LC material with $\Delta n=0.224, n_{\mathrm{e}}=1.741$, and $n_{\mathrm{o}}=1.517$ to $\lambda=550 \mathrm{~nm}$. The LC material parameter was the same as E7 (King Optronics Co. Ltd). The parameters related to the configuration are as follows: $R=55 \mu \mathrm{m}, l=120 \mu \mathrm{m}, g=10 \mu \mathrm{m}, w=10 \mu \mathrm{m}, s=5 \mu \mathrm{m}, d_{\mathrm{LC}}=30 \mu \mathrm{m}$, $\varepsilon_{\mathrm{LC}}=11.4$, and the thickness of the transparent ITO electrodes was $0.04 \mu \mathrm{m}$.

In the computer simulation process, we choose the anchoring energy force of the alignment layer as "strong". When the electric field intensity is stronger than the anchoring energy, the nematic LC director will rotate. Therefore, the anchoring energy may influence the LC director distribution. With the maturity of the alignment process, the thickness of the alignment layer can be as small as $0.1 \mu \mathrm{m}[31,32]$, so the depth of the frictional micro-groove is very shallow. Basically, only the surface of the alignment layer has a strong anchoring force. Furthermore, in our computer simulation, we used an ultra-low viscosity LC material LC layer, so the anchoring energy had little effect on the distribution of the nematic LC director and refractive index distribution.

We only researched the central $110 \mu \mathrm{m}$ (from $-55 \mu \mathrm{m}$ to $+55 \mu \mathrm{m}$ ) of the proposed model. Figure 2 shows the simulated electric potential distribution at $V_{\text {on }}=2.4 \mathrm{~V}_{\mathrm{rms}}, V_{\text {on }}=4.8 \mathrm{~V}_{\mathrm{rms}}, V_{\text {on }}=7.2 \mathrm{~V}_{\mathrm{rms}}$, respectively. The electric potential distribution area of individual LC microlens is framed by a dotted frame, as shown in Figure 2a. From Figure 2, we can see that the electric potential lines become denser from the center to the border of the LC microlens, so the electric field intensity becomes stronger, which means that the inclination angle of the LC director at the lens border is larger than that at the center. More significantly, the electric potential distribution is axisymmetric in space. However, 
for $V_{\text {on }}=2.4 \mathrm{~V}_{\mathrm{rms}}$, this voltage is too small for the LC director at the edge of the microlens to have a large tilt angle, resulting in a small refractive index difference and a relatively long focal length. Hence, we increased the voltage to $V_{\text {on }}=4.8 \mathrm{~V}_{\mathrm{rms}}$ in order to make the LC director on the microlens edge have a larger inclination angle. When $V_{\mathrm{on}}=4.8 \mathrm{~V}_{\mathrm{rms}}$, we can see that the electric potential lines at the edge of the lens become denser than $V_{\text {on }}=2.4 \mathrm{~V}_{\text {rms }}$, and the electric potential lines become smoother, which indicates that the gradient of the electric field distribution becomes better. But the operating voltage cannot be increased blindly. If we continue to increase the voltage to $V_{\text {on }}=7.2 \mathrm{~V}_{\mathrm{rms}}$, we can see from Figure $2 c$ that the gradient of the electric potential distribution in the microlens center becomes worse than that of $V_{\text {on }}=4.8 \mathrm{~V}_{\text {rms }}$, which implies that the phase profile at the microlens center will be flatter.

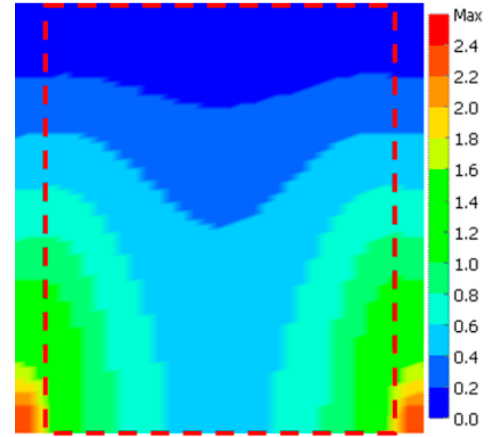

(a)

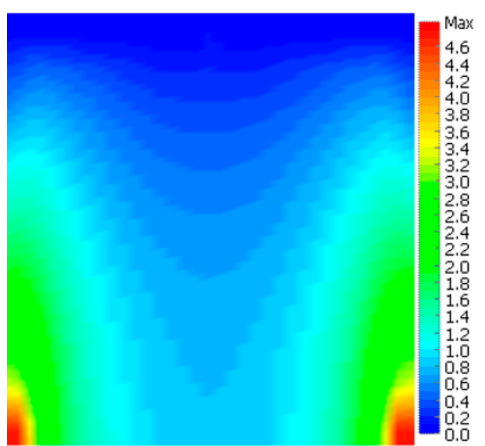

(b)

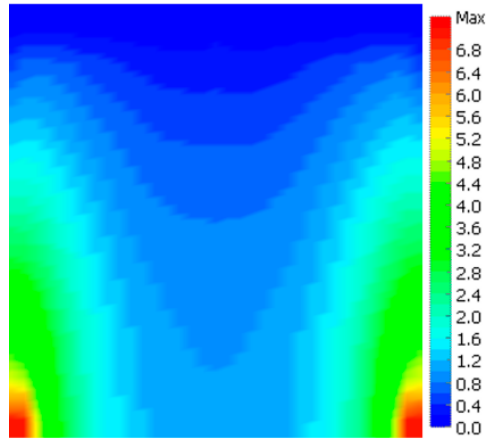

(c)

Figure 2. Simulated electric potential distribution within the LC bulk of the proposed model at (a) $V_{\text {on }}$ $=2.4 \mathrm{~V}_{\mathrm{rms}},\left(\right.$ b) $V_{\mathrm{on}}=4.8 \mathrm{~V}_{\mathrm{rms}}$, (c) $V_{\mathrm{on}}=7.2 \mathrm{~V}_{\mathrm{rms}}$, respectively (the area of individual LC microlens is framed by a red dotted frame in Figure $2 \mathrm{a})$.

Figure 3 shows the tilt angle distribution of the LC director of the proposed model at $V_{\text {on }}=4.8$ $\mathrm{V}_{\text {rms. }}$. From Figure 3a, we can see that the LC director in the LC bulk is rotated, while the LC director in the microlens center still remains horizontally aligned due to the existence of the central clearance between the common electrodes on the upper substrate and the gap between the strip electrodes. Due to the electric-field-induced LC director reorientation, the inclined angle distribution of the LC director changes gradually and is spatially symmetrical, as shown in Figure 3, which helps to generate an axisymmetric gradient refractive index distribution over the LC microlens aperture. In order to further study the LC orientation distribution within the nematic LC layer, we derived the top view of the LC director at $V_{\text {on }}=4.8 \mathrm{~V}_{\mathrm{rms}}$, as shown in Figure $3 \mathrm{~b}$. We could clearly see that the inclination angle of the LC director at the microlens border was larger than that at the center, which is very useful for obtaining a larger refractive index difference between the microlens center and border. It is noteworthy that the shortest focal length will be achieved when the LC director in the microlens border is almost completely aligned by the external electric field, while the LC director in the center is not yet reoriented due to the weaker electric field intensity. However, because of the anchoring energy force on the surface of the upper and lower substrates, it is not easy for the LC director at the microlens border to completely reorient. 


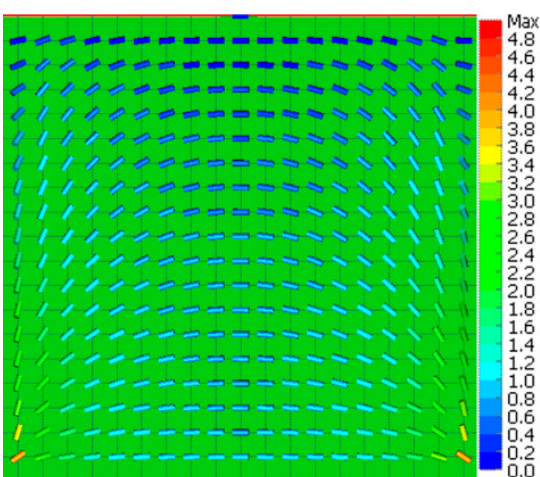

(a)

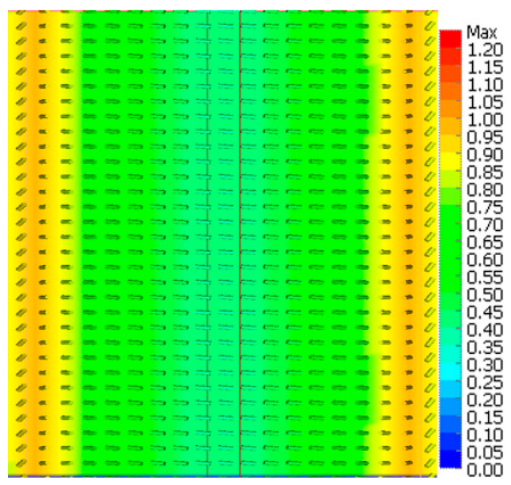

(b)

Figure 3. Simulated (a) cross section and (b) top view of the LC director across the nematic LC microlens at $V_{\text {on }}=4.8 \mathrm{~V}_{\text {rms }}$.

Figure 4 shows the simulated effective refractive index curve across the nematic LC microlens at $V_{\text {on }}=4.8 V_{\text {rms }}$. As shown in Figure 4, the effective refractive index curve of the incident linearly polarized wave is axisymmetric. The maximum effective refractive index was 1.7234 and the minimum effective refractive index was 1.5861. Therefore, the effective refractive index difference between the LC microlens border and center was 0.1371 . Given the relatively large effective refractive index difference across the LC layer, the focal length of this microlens will be very short.

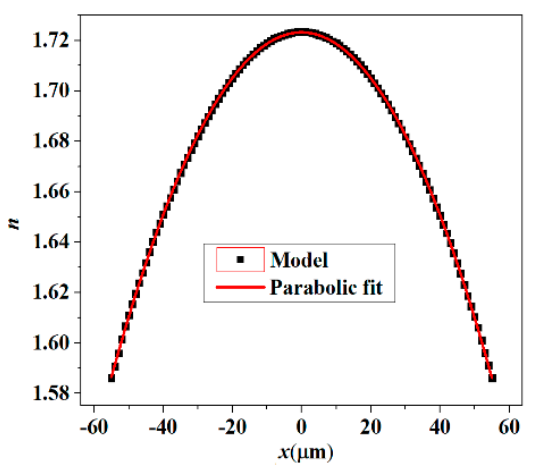

Figure 4. Simulated effective refractive index distribution curve across the LC microlens at $V_{\text {on }}=4.8 \mathrm{~V}_{\text {rms }}$.

Figure $5 \mathrm{a}-\mathrm{d}$ show the simulated refractive index distribution along the $x$ axis for the extraordinary ray of the proposed model at $V_{\text {on }}=3 \mathrm{~V}_{\mathrm{rms}}, V_{\text {on }}=4 \mathrm{~V}_{\mathrm{rms}}, V_{\mathrm{on}}=5 \mathrm{~V}_{\mathrm{rms}}$, and $V_{\text {on }}=6 \mathrm{~V}_{\mathrm{rms}}$, respectively. The refractive index distributions were axisymmetric under different operating voltages because of the symmetrical structure of the proposed LC microlens. When the operating voltage was $4 \mathrm{~V}_{\mathrm{rms}}$, the gradient of the refractive index distribution was better. When the operating voltage was $6 \mathrm{~V}_{\text {rms }}$, however, the refractive index distribution at both ends of the lens had almost no gradient change. Therefore, this part of the microlens has no focusing effect on the extraordinary ray, which means that the effective diameter of the microlens will become shorter. 


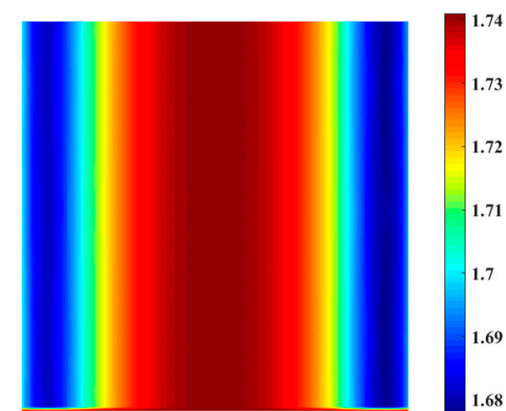

(a) $3 \mathrm{~V}_{\mathrm{rms}}$

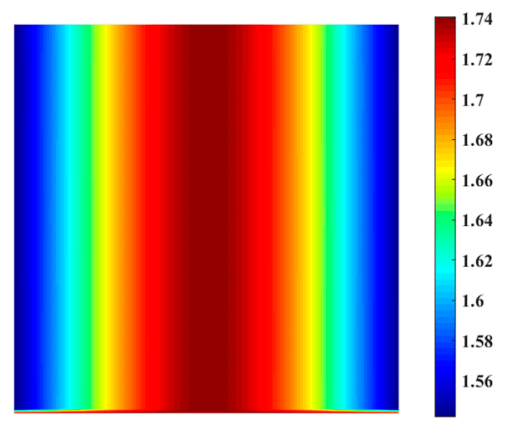

(c) $5 \mathrm{~V}_{\mathrm{rms}}$

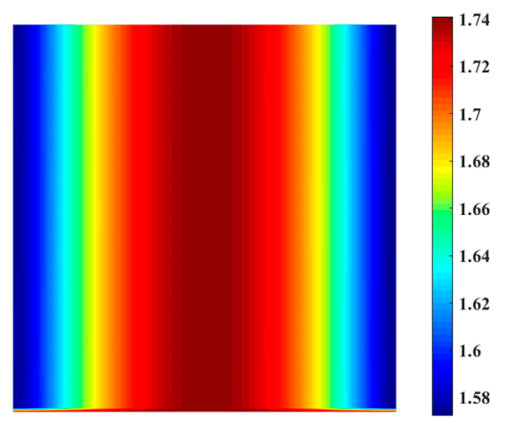

(b) $4 \mathrm{~V}$ rms

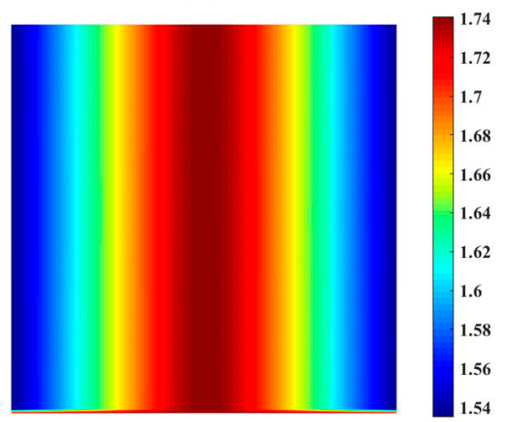

(d) $6 \mathrm{~V}_{\mathrm{rms}}$

Figure 5. Simulated top view of the refractive index distribution across the LC microlens along the $x$ axis for the extraordinary ray with different operating voltages.

Whether the phase profile is a parabola is an important criterion for evaluating the performance of a lens [33-36]. We plotted the profile of the relative phase difference across the LC microlens in the ideal alignment to study the focusing effect of the extraordinary ray, as shown in Figure 6 a. The ideal parabolic shape was also plotted in this figure. The relative phase difference distribution of the proposed model is represented by black curve with a red circle and the ideal parabolic shape is represented by the blue solid line. Here, for convenience, the relative phase difference of the LC microlens center was assumed to be zero. The form of the phase retardation for the proposed model implies that the LC microlens is a positive lens. The phase retardation between the microlens center and border for the proposed model was $14.978 \pi$. Compared to its relative phase difference profile with an ideal thin lens, we were surprised to find that they almost coincided, which means that such a LC microlens has almost no spherical aberration and can achieve great image quality. There are three reasons why the gap between common electrodes does not cause a non-smooth phase profile that affects image quality. First, the gap makes the central phase profile more ideal, which is designed to improve the imaging quality; second, the gap is in the center of a single microlens, and the gap of the left and right is a symmetrical structure; and finally, for each microlens, we only used one gap and two strip electrodes to obtain a microlens compared with the multi-strip electrode structure. One of the major challenges in fabricating this design is the alignment. Therefore, we also explored the relative phase profiles under different misalignment conditions between the top and bottom substrates at $V_{\text {on }}=4.8 \mathrm{~V}_{\text {rms }}$, as shown in Figure $6 \mathrm{~b}$. When the misalignment between the top and bottom substrates was from 0 to $5 \mu \mathrm{m}$, the center of the LC lens was a little biased, but it did not change much; moreover, the phase profile kept an ideal parabolic shape, which indicates that the proposed structure has great tolerance ability. 


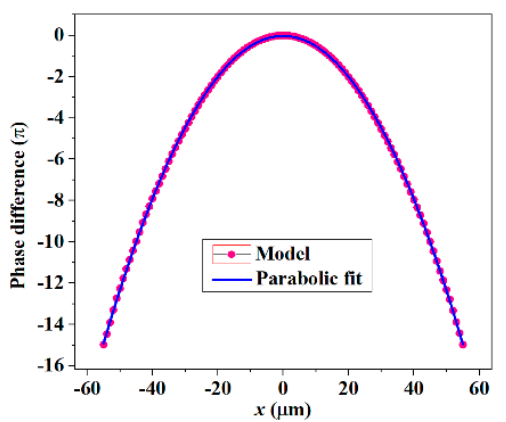

(a)

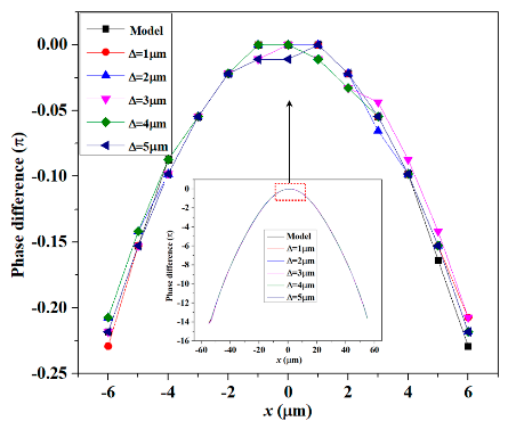

(b)

Figure 6. Simulated relative phase profiles of the LC microlens array (a) in an ideal alignment condition and (b) under different misalignment conditions at $V_{\text {on }}=4.8 \mathrm{~V}_{\mathrm{rms}}$.

To further discuss the optical characteristics of the proposed model with a simple electrode structure, with the other parameters unchanged, we changed the operating voltages from $4.8 \mathrm{~V}_{\mathrm{rms}}$ to $0.8 \mathrm{~V}_{\mathrm{rms}}$ to obtain the form of the phase retardation. As the operating voltage decreases, the relative phase profile across the LC microlens becomes flatter, which means that the focal length will increase, as shown in Figure 7. When the operating voltages were $4.8 \mathrm{~V}_{\mathrm{rms}}, 4.0 \mathrm{~V}_{\mathrm{rms}}, 3.2 \mathrm{~V}_{\mathrm{rms}}, 2.4 \mathrm{~V}_{\mathrm{rms}}, 1.6 \mathrm{~V}_{\mathrm{rms}}$ and $0.8 \mathrm{~V}_{\mathrm{rms}}$, the phase retardations of the center and border for the proposed LC microlens were $14.978 \pi, 13.047 \pi, 6.927 \pi, 1.789 \pi, 0.218 \pi$, and $\sim 0 \pi$, respectively.

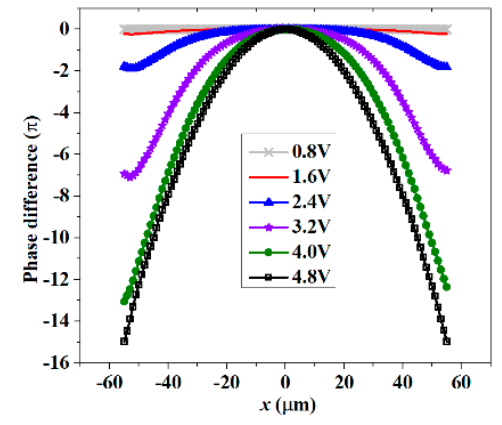

Figure 7. Simulated relative phase profiles across the LC microlens with different operating voltages.

The simulated voltage-dependent focal length of the LC microlens array with a simple electrode structure is shown in Figure 8a. From Figure 8a, we can see that the focal length of the LC microlens decreased as the operating voltage increased. When the operating voltage was sufficiently high, the LC director was reoriented along the electric field direction. Hence, it can be concluded from Equation (1) that the effective refractive index is reduced at the microlens border while it remains unchanged at the microlens center. From Equation (2), with the increase of $\delta n$, the focal length becomes shorter. When the voltages were $1.6 \mathrm{~V}_{\mathrm{rms}}, 2.4 \mathrm{~V}_{\mathrm{rms}}, 4.0 \mathrm{~V}_{\mathrm{rms}}$ and $4.8 \mathrm{~V}_{\mathrm{rms}}$, the focal lengths were $24.008 \mathrm{~mm}$, $3.019 \mathrm{~mm}, 0.445 \mathrm{~mm}$, and $0.381 \mathrm{~mm}$, respectively. Figure $8 \mathrm{~b}$ shows the simulated response time curve for the LC microlens at $V_{\text {on }}=4.8 \mathrm{~V}_{\text {rms }}$. The dynamic response time study was conducted by placing the LC cell between crossed polarizers with its rubbing direction $90^{\circ}$ to it. The rise time was $209.83 \mathrm{~ms}$ and the decay time was $128.87 \mathrm{~ms}$. Such a dynamic response time is relatively fast during the focus change because of the low rotational viscosity of the LC material and the relatively thin cell thickness. This designed microlens array is mainly suitable for $2 \mathrm{D} / 3 \mathrm{D}$ switching in high resolution displays. It is close to the display screen and has little stray light, so the off-axis phenomenon will not be obvious. If the LC microlens array is used for 2D/3D switching, the dynamic response time needs to be further improved. In addition to adopting low viscosity LC materials, we can apply an overdriven and undershoot driving scheme to improve the response time. Moreover, the proposed electrically tunable LC microlenses is not polarization independent. To resolve this challenging issue, we can use two homogeneous LC layers with orthogonal rubbing directions. 


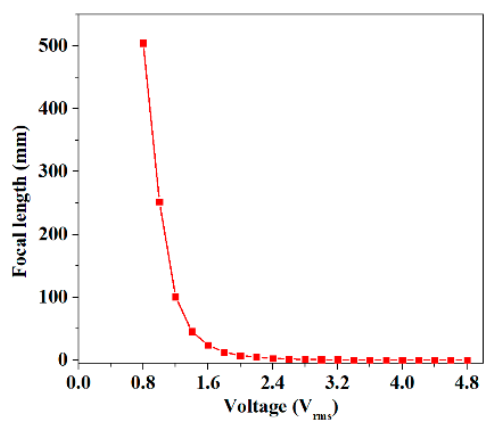

(a)

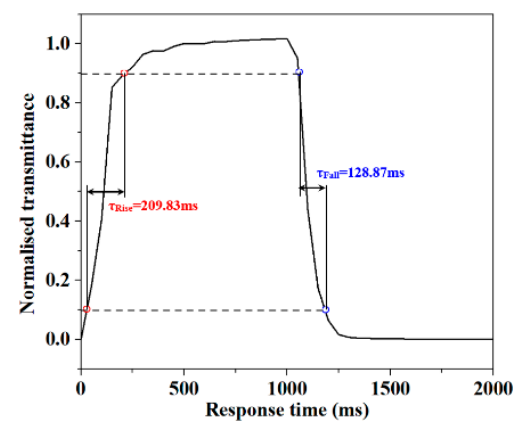

(b)

Figure 8. Simulated voltage-dependent focal length and response time curve of the proposed model.

\section{Materials and Methods}

The parameters of the E7-LC material used in the simulation are as follows: elastic constant $K_{11}=11.7 \mathrm{pN}, K_{22}=8.8 \mathrm{pN}, K_{33}=19.5 \mathrm{pN}$, refractive index of $o$ and $e$ waves $n_{\mathrm{e}}=1.741, n_{\mathrm{o}}=1.517$, and dielectric anisotropy $\Delta \varepsilon=11.4$.

To validate the performance of the proposed LC microlens array, we carried out electro-optical simulation using the commercial simulation software TechWiz LCD 3D (Sanayi System Co. Ltd., Incheon, Korea).

\section{Conclusions}

We proposed an electrically tunable-focusing LC microlens array with a simple electrode structure. In the presented approach, a certain gap between the top common electrodes was designed. This electrode gap between common electrodes is the distinguishing part of this structure when compared with conventional LC lenses. When a voltage is applied in the center of the lens, an ideal gradient electric field intensity is generated between the gap part and the lower substrate, which helps maintain the LC horizontal orientation. Meanwhile, for each microlens, we only used two strip electrodes to achieve a very perfect phase profile compared with the multi-strip electrode structure. The optimized LC microlens array offers good performance including a wide focusing range, light weight, low operating voltage, relatively thin cell thickness, and almost no color dispersion. Moreover, this kind of lens is fairly easy to fabricate given its simple electrode structure. Its applications in photonic microdevices are foreseeable.

Author Contributions: L.-L.T. and Q.-H.W. conceived the original idea and wrote the paper; F.C. and H.D. analyzed the data; L.L. performed the analysis.

Funding: The work was supported by the National Natural Science Foundation of China under Grant No. 61535007 and the Equipment Research Program in Advance of China under Grant No. JZX2017-1570/Y464.

Conflicts of Interest: The authors declare no conflicts of interest.

\section{References}

1. Radtke, D.; Duparré, J.; Dannberg, P. Implementation of field lens arrays in beam-deflecting microlens array telescopes. Appl. Opt. 2004, 43, 4854-4861.

2. Smith, P.J.; Taylor, C.M.; Mccabe, E.M.; Selviah, D.R.; Day, S.E.; Commander, L.G. Switchable fiber coupling using variable-focal-length microlenses. Rev. Sci. Instrum. 2001, 72, 3132-3134. [CrossRef]

3. Fu, Y.; Bryan, N. Design of hybrid micro-diffractive-refractive optical element with wide field of view for free space optical interconnections. Opt. Express 2002, 10, 540-549. [CrossRef] [PubMed]

4. Duparré, J.; Götz, B.; Göring, R. Micro-optical $1 \times 4$ fiber switch for multimode fibers with $600-\mu \mathrm{m}$ core diameters. Appl. Opt. 2003, 42, 6889-6896. [CrossRef] [PubMed]

5. Igalstian, T.; Asatryan, K.; Presniakov, V.; Zohrabyan, A. Electrically variable liquid crystal lenses for ophthalmic distance accommodation. Opt. Express 2019, 27, 18803-18817. [CrossRef] [PubMed] 
6. Xiong, Z.L.; Wang, Q.H.; Li, S.L.; Deng, H.; Ji, C.C. Partially-overlapped viewing zone based integral imaging system with super wide viewing angle. Opt. Express 2014, 22, 22268-22277. [CrossRef] [PubMed]

7. Luo, L.; Wang, Q.H.; Xing, Y.; Deng, H.; Ren, H.; Li, S. 360-degree viewable tabletop 3D display system based on integral imaging by using perspective-oriented layer. Opt. Commun. 2019, 438, 54-60. [CrossRef]

8. Huang, Y.P.; Chen, C.W.; Shen, T.C. High Resolution Autostereoscopic 3D Display with Scanning Multi-Electrode Driving Liquid Crystal (MeD-LC) Lens. Dig. Tech. Pap. 2009, 25, 336-339. [CrossRef]

9. Hong, H.K.; Jung, S.M.; Lee, B.J.; Shin, H.H. Electric-field-driven LC lens for 3D/2D autostereoscopic display. J. Soc. Inf. Disp. 2012, 17, 399-406. [CrossRef]

10. Kim, S.U.; Na, J.H.; Kim, C.; Lee, S.D. Design and fabrication of liquid crystal-based lenses. Liq. Cryst. 2017, 44, 1-12. [CrossRef]

11. Wang, Y.J.; Lin, Y.H. An optical system for augmented reality with electrically tunable optical zoom function and image registration exploiting liquid crystal lenses. Opt. Express 2019, 27, 21163-21172. [CrossRef]

12. Chou, P.Y.; Wu, J.Y.; Huang, S.H.; Wang, C.P.; Qin, Z.; Huang, C.T.; Hsieh, P.Y.; Lee, H.H.; Lin, T.H.; Huang, Y.P. Hybrid light field head-mounted display using time-multiplexed liquid crystal lens array for resolution enhancement. Opt. Express 2019, 27, 1164-1178. [CrossRef] [PubMed]

13. Ye, M.; Chen, X.; Li, Q.; Zeng, J.; Yu, S. Depth from defocus measurement method based on liquid crystal lens. Opt. Express 2019, 26, 28413-28420. [CrossRef] [PubMed]

14. Algorri, J.F.; Bennis, N.; Urruchi, V.; Morawiak, P.; Jaroszewicz, L.R. Tunable liquid crystal multifocal microlens array. Sci. Rep. 2017, 7, 17318. [CrossRef] [PubMed]

15. Ye, M. Liquid crystal lens with a focal length that is variable in a wide range. Appl. Opt. 2004, 43, 6407-6412. [CrossRef] [PubMed]

16. Huang, Y.P.; Liao, L.Y.; Chen, C.W. 2-D/3-D switchable autostereoscopic display with multi-electrically driven liquid-crystal (MeD-LC) lenses. J. Soc. Inf. Disp. 2010, 18, 642-646. [CrossRef]

17. Ye, M.; Hayasaka, S.; Sato, S. Liquid crystal lens array with hexagonal-hole-patterned electrodes. Jpn. J. Appl. Phys. 2004, 43, 6108-6111. [CrossRef]

18. Lin, Y.H.; Chen, H.S. Electrically tunable-focusing and polarizer-free liquid crystal lenses for ophthalmic applications. Opt. Express 2013, 21, 9428-9436. [CrossRef] [PubMed]

19. Asatryan, K.; Presnyakov, V.; Tork, A.; Zohrabyan, A.; Bagramyan, A.; Galstian, T. Optical lens with electrically variable focus using an optically hidden dielectric structure. Opt. Express 2010, 18, 13981-13992. [CrossRef] [PubMed]

20. Cui, J.P.; Fan, H.X.; Wang, Q.H. A polarisation-independent blue-phase liquid crystal microlens using an optically hidden dielectric structure. Liq. Cryst. 2017, 44, 643-647. [CrossRef]

21. Lin, Y.H.; Chen, H.S.; Lin, H.C.; Tsou, Y.S.; Hsu, H.K.; Li, W.Y. Polarizer-free and fast response microlens arrays using polymer-stabilized blue phase liquid crystals. Appl. Phys. Lett. 2010, 96, 113505. [CrossRef]

22. Chu, F.; Dou, H.; Li, G.P.; Song, Y.L.; Li, L.; Wang, Q.H. A polarisation-independent blue-phase liquid crystal lens array using gradient electrodes. Liq. Cryst. 2017, 45, 715-720. [CrossRef]

23. Li, R.; Chu, F.; Dou, H.; Tian, L.L.; Hou, W.Y.; Li, L.; Wang, Q.H. A blue-phase liquid crystal lens array based on dual square ring-patterned electrodes. Liq. Cryst. 2019, 46, 1266-1272. [CrossRef]

24. Li, Y.; Huang, S.; Zhou, P.; Liu, S.; Lu, J.; Li, X.; Su, Y. Polymer-Stabilized Blue Phase Liquid Crystals for Photonic Applications. Adv. Mater. Technol. 2016, 1, 1600102. [CrossRef]

25. Hisakado, Y.; Kikuchi, H.; Nagamura, T.; Kajiyama, T. Large electro-optic Kerr effect in polymer-stabilized liquid-crystalline blue phases. Adv. Mater. 2005, 17, 96-98. [CrossRef]

26. Lin, Y.H.; Wang, Y.J.; Reshetnyak, V. Liquid crystal lenses with tunable focal length. Liq. Cryst. Rev. 2017, 5, 111-143. [CrossRef]

27. Li, L.; Bryant, D.; Bos, P.J. Liquid crystal lens with concentric electrodes and inter-electrode resistors. Liq. Cryst. Rev. 2014, 2, 130-154. [CrossRef]

28. Lin, Y.H.; Chen, H.S.; Chen, M.S. Electrically tunable liquid crystal lenses and applications. Mol. Cryst. Liq. Cryst. 2014, 596, 12-21. [CrossRef]

29. Wang, B.; Ye, M.; Honma, M.; Nose, T.; Sato, S. Liquid crystal lens with spherical electrode. Jpn. J. Appl. Phys. 2014, 41, 1232-1233. [CrossRef]

30. Ren, H.; Wu, S.T. Adaptive liquid crystal lens with large focal length tenability. Opt. Express 2006, 14, 11292-11298. [CrossRef] 
31. He, Z.Q.; Lee, Y.H.; Chen, Y.; Chanda, D.; Wu, S.T. Switchable Pancharatnam-Berry microlens array with nano-imprinted liquid crystal alignment. Opt. Lett. 2018, 43, 5062-5065. [CrossRef]

32. He, Z.; Lee, Y.H.; Gou, F.; Franklin, D.; Chanda, D.; Wu, S.T. Polarization-independent phase modulators enabled by two-photon polymerization. Opt. Express 2017, 25, 33688-33694. [CrossRef]

33. Lee, C.T.; Li, Y.; Lin, H.Y.; Wu, S.T. Design of polarization-insensitive multi-electrode GRIN lens with a blue-phase liquid crystal. Opt. Express 2011, 19, 17402-17407. [CrossRef]

34. Li, Y.; Wu, S.T. Polarization independent adaptive microlens with a blue-phase liquid crystal. Opt. Express 2011, 19, 8045-8050. [CrossRef]

35. Li, Y.; Liu, Y.F.; Li, Q.; Wu, S.T. Polarization independent blue-phase liquid crystal cylindrical lens with a resistive film. Appl. Opt. 2012, 51, 2568-2572. [CrossRef]

36. Kao, Y.Y.; Chao, P.C.P.; Hsueh, C.W. A new low-voltage-driven GRIN liquid crystal lens with multiple ring electrodes in unequal widths. Opt. Express 2010, 18, 18506-18518. [CrossRef]

(C) 2019 by the authors. Licensee MDPI, Basel, Switzerland. This article is an open access article distributed under the terms and conditions of the Creative Commons Attribution (CC BY) license (http://creativecommons.org/licenses/by/4.0/). 